\title{
Long-Term Functional Outcome for Patients Treated under Drip and Stay versus Drip and Ship Paradigm: A Single Network Experience
}

\author{
Sami Al Kasab, ${ }^{\mathrm{a}, *}$ Eyad Almallouhi, ${ }^{\mathrm{b}, *}$ Jillian B. Harvey, ${ }^{\mathrm{c}}$ Tarun Girotra, ${ }^{\mathrm{d}}$ Ellen Debenham, ${ }^{\mathrm{b}}$ \\ Nancy Turner, ${ }^{b}$ Christine A. Holmstedt ${ }^{b}$ \\ ${ }^{a}$ Department of Neurology, University of lowa, lowa City, IA, USA \\ ${ }^{b}$ Department of Neurology, Medical University of South Carolina, Charleston, SC, USA \\ 'Department of Healthcare Leadership and Management, College of Health Professions, Medical University of South Carolina, Charleston, SC, USA \\ ${ }^{d}$ Department of Neurology, University of New Mexico, Albuquerque, NM, USA
}

*These authors contributed equally to the manuscript as first author.

\section{Dear Sir:}

The implementation of telestroke networks significantly improved access to intravenous tissue plasminogen activator (IVtPA) in rural areas. ${ }^{1}$ Through the telestroke system of care, patients who present to a community hospital (spoke) are evaluated using a videoconferencing platform by a stroke expert. Telestroke patients who receive IV-tPA either remain at the spoke (drip and stay) or are transferred to a tertiary medical center (drip and ship). ${ }^{2}$

We reviewed the prospectively collected data on all consecutive telestroke patients who received IV-tPA through the Medical University of South Carolina (MUSC) telestroke network between January 2016 and March 2018. Details on the MUSC telestroke network were described before. ${ }^{3}$ Some spoke sites are able to keep stroke patients after receiving tPA (drip and stay), while other sites transfer all patients who receive tPA to a comprehensive stroke center (drip and ship). Drip and ship patients who were transferred to the hub for mechanical thrombectomy evaluation because of clinical suspicion for large vessel occlusion were excluded from this analysis. Our primary outcome was the modified Rankin Scale (mRS) at 90 days. A good functional outcome was defined as a 90 -day $m R S$ of $0-2$.

We assessed the predictors of having good 90-day outcome using a generalized linear model (GLM) with logit link control- ling for age, sex, race, door to needle time and baseline National Institutes of Health Stroke Scale (NIHSS). Sensitivity analysis was done using worst-case scenario method to account for patients who were excluded from the GLM because of missing 90day mRS ( 81 in the drip and stay group and 19 in the drip and ship group). This study was approved by the Institutional Review Board of the Medical University of South Carolina, with the need for written informed consent waived.

During the study period, 797 patients received tPA at spoke sites through MUSC telestroke network. Two hundred and eighty patients were excluded for missing data or because they were transferred to a thrombectomy-capable center for thrombectomy evaluation (Figure 1). A total of 517 patients were included in the analysis; 410 (79.3\%) in the drip and stay group, and 107 (20.7\%) in the drip and ship group. As shown in Table 1, there was no difference baseline NIHSS or door to needle time in both groups. A 90-day good functional outcome was achieved in 323 patients $(78.8 \%)$ in the drip and stay and in 80 patients $(74.8 \%)$ in the drip and ship group ( $P=0.372)$ (Figure 2). On multivariate analysis, we found no difference in the adjusted relative risk (RR) of having 90-day $m R S \leq 2$ between drip and ship and drip and stay patients (RR, 0.95; 95\% confidence interval $[\mathrm{Cl}], 0.85$ to $1.10 ; P=0.365$ ) (Table 2). Similar results were found on sensitivity analysis using worst case scenario to account for patients with missing 90-day $\mathrm{mRS}(\mathrm{RR}, 0.96 ; 95 \% \mathrm{Cl}, 0.83$ to $1.11 ; P=0.572)$. 


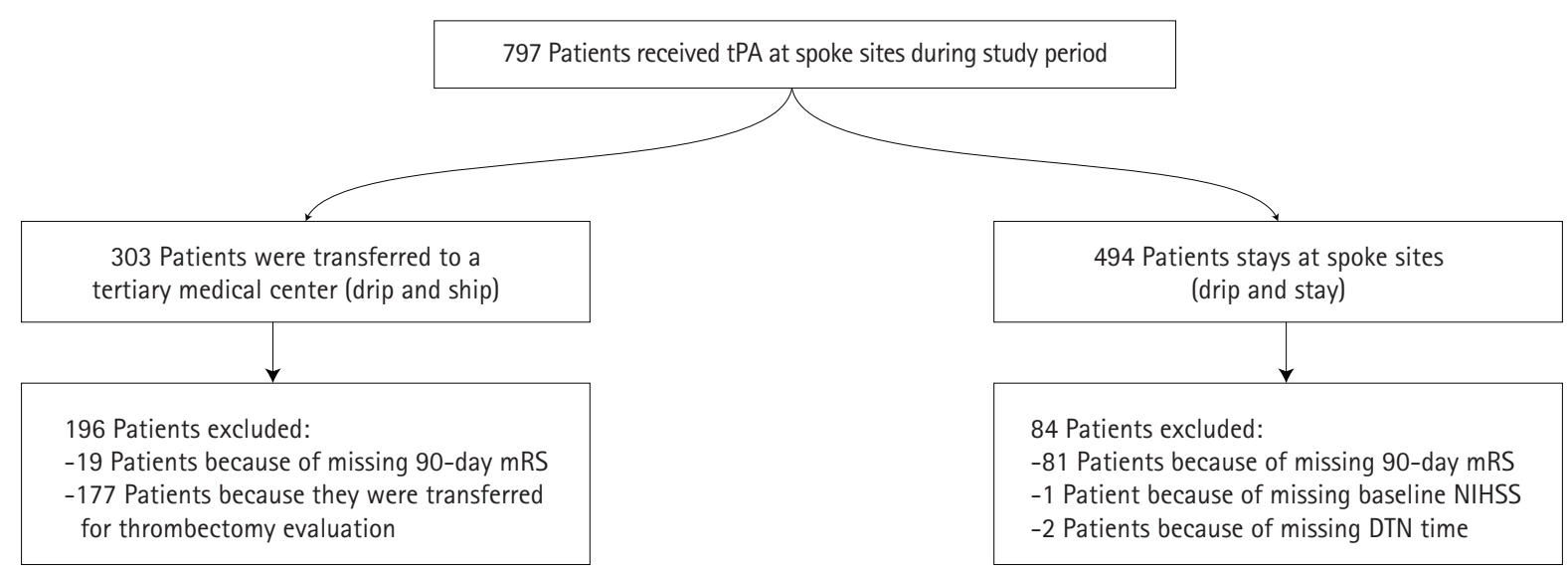

Figure 1. Study flowchart. tPA, tissue plasminogen activator; mRS, modified Rankin Scale; NIHSS, National Institutes of Health Stroke Scale; DTN, door to needle.

Table 1. Characteristics of study cohort

\begin{tabular}{|c|c|c|c|c|}
\hline Variable & Drip and stay group $(n=410)$ & Drip and ship group $(n=107)$ & Total $(n=517)$ & $P$ \\
\hline Age (yr) & $66.1 \pm 16.3$ & $66.7 \pm 15.4$ & $66.3 \pm 16.1$ & 0.746 \\
\hline Sex & & & & 0.013 \\
\hline Female & $246(60.0)$ & $50(46.7)$ & $296(57.3)$ & \\
\hline Male & $164(40.0)$ & $57(53.3)$ & $221(42.8)$ & \\
\hline Race & & & & 0.830 \\
\hline Black & $110(26.8)$ & $28(26.2)$ & $138(26.7)$ & \\
\hline White & $265(64.6)$ & $72(67.3)$ & $337(65.2)$ & \\
\hline Other & 35 (8.54) & $7(6.5)$ & $42(8.1)$ & \\
\hline Admission NIHSS & $5(4-8)$ & $6(5-9)$ & $5(4-8)$ & 0.166 \\
\hline Door to needle time* & $56(43-73)$ & $58(25-140)$ & $57(43-75)$ & 0.465 \\
\hline $\mathrm{sICH}$ & $7(1.7)$ & $3(2.8)$ & $10(1.9)$ & 0.460 \\
\hline
\end{tabular}

Values are presented as mean \pm standard deviation, number (\%), or median (interquartile range).

NIHSS, National Institutes of Health Stroke Scale; sICH, symptomatic intracerebral hemorrhage.

${ }^{*}$ Measured in minutes; ${ }^{+S}$ ymptomatic hemorrhagic transformation defined using the European Australian Cooperative Acute Stroke Study II (ECAS II) definition.

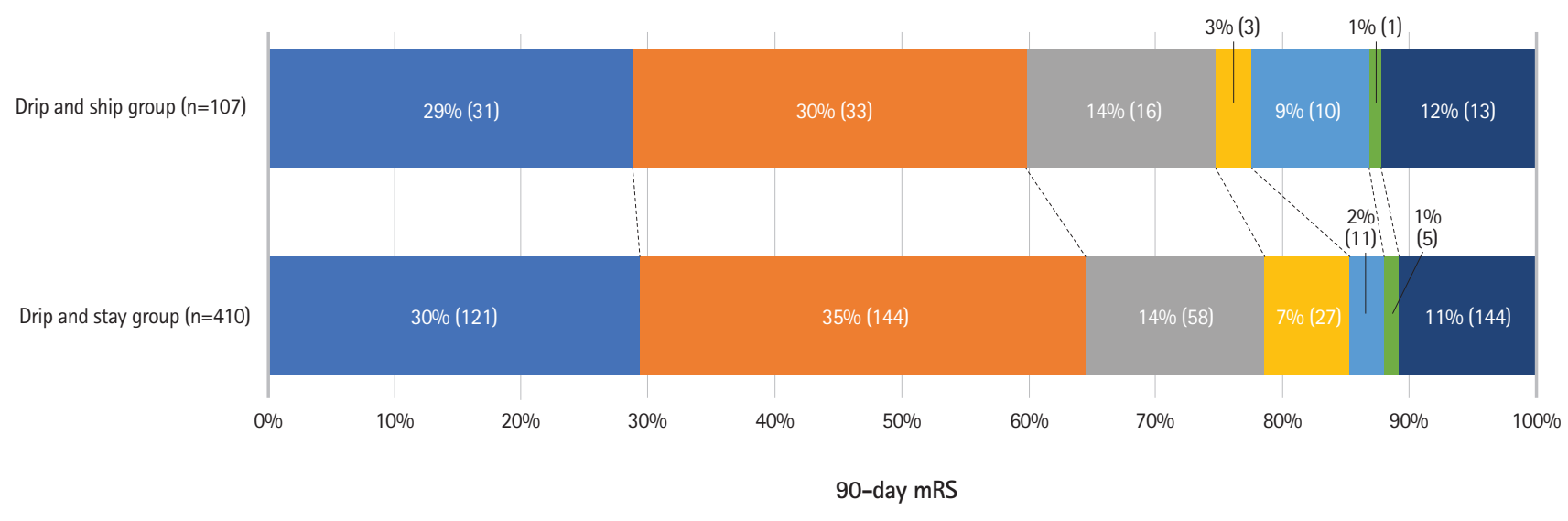

\begin{tabular}{l|l|l|l|l|l:l}
0 & 1 & 2 & 3 & 4 & -5
\end{tabular}

Figure 2. A 90-day modified Rankin Scale (mRS) distribution in both groups. 
Table 2. Results of the generalized regression model to predict good 90-day functional outcomes

\begin{tabular}{lccc}
\hline Outcome & Relative risk & $95 \% \mathrm{Cl}$ & $P$ \\
\hline Drip-and-ship & 0.95 & $0.85-1.10$ & 0.365 \\
Baseline NIHSS & 0.94 & $0.93-0.96$ & 0.007 \\
Door to needle time & 1.00 & $0.99-1.01$ & 0.861 \\
Age & 0.99 & $0.99-0.99$ & 0.001 \\
Female & 0.99 & $0.92-1.06$ & 0.947 \\
White race & 1.11 & $0.98-1.24$ & 0.112 \\
\hline
\end{tabular}

$\mathrm{Cl}$, confidence interval; NIHSS, National Institutes of Health Stroke Scale.

Our study shows that patients treated with intravenous alteplase through telestroke and who otherwise do not require a higher level of care can remain at spoke centers and can have excellent outcomes that are comparable to those transferred to a tertiary medical center.

Early experience with tPA demonstrated that mortality was higher when tPA was administered in inexperienced hospitals, which led to more support for telestroke networks to be able to provide a safe and efficient solution for acute stroke management. ${ }^{4,5}$ With more evidence emerging concerning the benefits of telestroke consultations, including higher rates of thrombol$y s i{ }^{6}{ }^{6}$ one of the main questions remaining is whether stroke patients post-tPA will experience better outcomes if they stay at the spoke sites (drip and stay) or if they are transferred to a tertiary medical center (drip and ship). This question is especially important for patients who are not candidates for endovascular or neurosurgical intervention. A few studies in the past have tried to answer this question, including a study by Yaghi et al. ${ }^{7}$ which demonstrated that telestroke patients with mild stroke (NIHSS $<8$ ) have similar long-term outcomes whether they stay at a spoke or are transferred to a primary stroke center. However, patients with moderate to severe stroke (NIHSS $\geq 8$ ) had higher odds of having a good long-term outcome if they are transferred to the telestroke hub. ${ }^{7}$ Our study has shown similar results with an advantage that the comparison groups were more homogeneous in terms of stroke severity given that all patients with suspected or confirmed large vessel occlusion were excluded from the study.

After the extension of the mechanical thrombectomy treatment window, more patients are expected to be transferred to thrombectomy-capable centers for thrombectomy evaluation and treatment. ${ }^{8}$ Thus, new strategies should be put in place to ensure that only patients who will benefit from the transfer are being transferred. With the cumulative evidence that has shown that telestroke patients may have good outcomes when they remain at spoke sites following thrombolysis, ${ }^{5,9}$ more efforts should be taken to ensure that patients who are treated under the drip and stay paradigm receive equivalent care as patients who are treated under the drip and ship paradigm.

Our study has a few limitations. First, although the telestroke $\log$ at MUSC is prospectively maintained, the study parameters were reviewed retrospectively. Second, 90-day mRS was missing for 100 patients. However, sensitivity analysis using worst case scenario showed similar results to the primary multivariate analysis. Finally, we were unable to control for factors that affected the decision for transfer to a tertiary medical center including spoke hospital staffing, presentation during off-hours and other medical complications.

\section{References}

1. Zhang D, Wang G, Zhu W, Thapa JR, Switzer JA, Hess DC, et al. Expansion of telestroke services improves quality of care provided in super rural areas. Health Aff (Millwood) 2018;37: 2005-2013.

2. Heffner DL, Thirumala PD, Pokharna P, Chang YF, Wechsler L. Outcomes of spoke-retained telestroke patients versus hubtreated patients after intravenous thrombolysis: telestroke patient outcomes after thrombolysis. Stroke 2015;46:3161-3167.

3. Al Kasab S, Adams RJ, Debenham E, Jones DJ, Holmstedt CA. Medical University of South Carolina Telestroke: a telemedicine facilitated network for stroke treatment in South Carolina-a progress report. Telemed J E Health 2017;23:674-677.

4. Heuschmann PU, Kolominsky-Rabas PL, Roether J, Misselwitz B, Lowitzsch K, Heidrich J, et al. Predictors of in-hospital mortality in patients with acute ischemic stroke treated with thrombolytic therapy. JAMA 2004;292:1831-1838.

5. Schwab S, Vatankhah B, Kukla C, Hauchwitz M, Bogdahn U, Fürst $A$, et al. Long-term outcome after thrombolysis in telemedical stroke care. Neurology 2007;69:898-903.

6. Müller-Barna P, Schwamm LH, Haberl RL. Telestroke increases use of acute stroke therapy. Curr Opin Neurol 2012;25:5-10.

7. Yaghi S, Harik SI, Hinduja A, Bianchi N, Johnson DM, Keyrouz SG. Post t-PA transfer to hub improves outcome of moderate 
to severe ischemic stroke patients. J Telemed Telecare 2015;21: 396-399.

8. Nogueira RG, Jadhav AP, Haussen DC, Bonafe A, Budzik RF, Bhuva $P$, et al. Thrombectomy 6 to 24 hours after stroke with a mismatch between deficit and infarct. N Engl J Med 2018;378:11-21.

9. Almallouhi E, Holmstedt CA, Harvey J, Reardon C, Guerrero WR, Debenham $E_{1}$ et al. Long-term functional outcome of telestroke patients treated under drip-and-stay paradigm compared with patients treated in a comprehensive stroke center: a single center experience. Telemed J E Health 2018 Sep 29 [Epub]. https://doi.org/10.1089/tmj.2018.0137.
Correspondence: Eyad Almallouhi

Department of Neurology, Medical University of South Carolina, 96 Jonathan Lucas Street, Charleston, SC 29425, USA

Tel: +1-843-792-1696

Fax: +1-843-792-2317

E-mail:Almallou@musc.edu

Received: February 28, 2019

Revised: March 20, 2019

Accepted: March 20, 2019

The authors have no financial conflicts of interest. 